\title{
Influence of Lorentz violation on Dirac quasinormal modes in the Schwarzschild black hole spacetime
}

\author{
Songbai Chen* \\ Department of Physics, Fudan University, Shanghai 200433, P. R. China \\ Institute of Physics and Department of Physics, \\ Hunan Normal University, Changsha, Hunan 410081, P. R. China \\ Bin Wang $\dagger$ \\ Department of Physics, Fudan University, Shanghai 200433, P. R. China \\ Rukeng $\mathrm{Su}$ 主 \\ China Center of Advanced Science and Technology (World Laboratory), \\ P.B.Box 8730, Beijing 100080, Peoples Republic of China \\ Department of Physics, Fudan University, Shanghai 200433, P. R. China
}

\begin{abstract}
Using the third-order WKB approximation and monodromy methods, we investigate the influence of Lorentz violating coefficient $b$ (associated with a special axial-vector $b_{\mu}$ field ) on Dirac quasinormal modes in the Schwarzschild black hole spacetime. At fundamental overtone, the real part decreases linearly as the parameter $b$ increases. But the variation of the imaginary part with $b$ becomes more complex. For the larger multiple moment $k$, the magnitude of imaginary part increases with the increase of $b$, which means that presence of Lorentz violation makes Dirac field damps more rapidly. At high overtones, it is found that the real part of high-damped quasinormal frequency does not tend to zero, which is quite a different from the asymptotic Dirac quasinormal modes without Lorentz violation.

PACS numbers: 04.30.-w, 04.62.+v, 97.60.Lf
\end{abstract}

*Electronic address: csb3752@hotmail.com

†Electronic address: wangb@fudan.edu.cn

${ }^{\ddagger}$ Electronic address: rksu@fudan.ac.cn 


\section{INTRODUCTION}

Since Lorentz invariance was discovered, it has been great of importance in many fields of the fundamental physics, such as the Einstein's special relativity theory, particle physics and high energy physics. However, the recent development of unified gauge theories and the observation of high energy cosmic rays [1] [2] [3] imply that Lorentz symmetry is only an approximate symmetry of nature and may be spontaneously broken in the more fundamental physics defined in a higher scale of energy. Obviously, the existence of Lorentz violation will make a great influence on the fundamental physics and lead to many subjects need to be reconsidered. Therefore, a great deal of effort has been attracted to study Lorentz violation in the different fields [4]- 22].

One of interesting theory models with Lorentz violation is the Standard Model Extension[8] [9] [10]. It offers a consistent theoretical framework which includes the standard model and allows for small violations of Lorentz and CPT symmetry. This small spontaneous breaking of Lorentz symmetry maybe arise from the presence of nonzero vacuum expectation values for Lorentz tensors defined in an underlying theory. A straightforward method of implementing Lorentz violation in the curve spacetime is to imagine the existence of a tensor field with a non-vanishing expectation value and couple this tensor to gravity or matter fields. In gravitational theories, vierbein formalism is used widely because it can build a link between the covariant components $T_{\mu \nu \ldots}$ of a tensor field in a coordinate basis and the corresponding covariant components $T_{a b \ldots}$ of the tensor field in a local Lorentz frame. The link can be described by $T_{\mu \nu \cdots}=e^{a}{ }_{\mu} e^{b}{ }_{\nu} \cdots T_{a b \cdots}$, where vierbein $e^{a}{ }_{\mu}$ is defined by $g_{\mu \nu}=\eta_{a b} e^{a}{ }_{\mu} e_{\nu}^{a}$. Moreover, it is also found that vierbein formalism can deal with local Lorentz transformation and diffeomorphisms, which are two basic types of spacetime transformations in gravitational theories. Bluhm and Kostelecky[11] make use of this tool and find that any violation of diffeomorphism invariance via vacuum values of vectors or tensors breaks local Lorentz invariance. The converse is also true. For example, if a spacetime vector $b_{\mu}$ acquires a fixed vacuum expectation value $\left\langle b_{\mu}\right\rangle$, which breaks diffeomorphisms, then the associated local vector $b_{a}$ as given by contraction with the inverse of vierbein also acquires a fixed vacuum expectation value $\left\langle b_{a}\right\rangle$. The presence of quantity $\left\langle b_{a}\right\rangle$ breaks local Lorentz symmetry. Therefore, it is natural for us to adopt vierbein formalism to study of Lorentz violation in the curve spacetime.

Adopting vierbein formalism, the fermion partial action $S_{\psi}$ in Standard Model Extension can be explicitly expressed as

$$
S_{\psi}=\int d^{4} x \sqrt{-g}\left(\frac{1}{2} i e_{a}^{\mu} \bar{\psi} \Gamma^{a} \overleftrightarrow{D_{\mu}} \psi-\bar{\psi} M^{*} \psi\right)
$$


where $e^{\mu}{ }_{a}$ is the inverse of the vierbein $e^{a}{ }_{\mu}$. The symbols $\Gamma^{a}$ and $M^{*}$ are

$$
\Gamma^{a} \equiv \gamma^{a}-c_{\mu \nu} e^{\nu a} e^{\mu}{ }_{b} \gamma^{b}-d_{\mu \nu} e^{\nu a} e^{\mu}{ }_{b} \gamma_{5} \gamma^{b}-e_{\mu} e^{\mu a}-i f_{\mu} e^{\mu a} \gamma_{5}-\frac{1}{2} g_{\lambda \mu \nu} e^{\nu a} e^{\lambda}{ }_{b} e^{\mu} \sigma^{b c},
$$

and

$$
M^{*} \equiv m+i m_{5} \gamma_{5}+a_{\mu} e^{\mu}{ }_{a} \gamma^{a}+b_{\mu} e_{a}^{\mu} \gamma_{5} \gamma^{a}+\frac{1}{2} H_{\mu \nu} e_{a}^{\mu} e_{b}{ }_{b} \sigma^{a b}
$$

The first terms of Eqs.(2) and (3) lead to the usual Lorentz invariant kinetic term and mass for the Dirac field. The parameters $a_{\mu}, b_{\mu}, c_{\mu \nu}, d_{\mu \nu}, e_{\mu}, f_{\mu}, g_{\lambda \mu \nu}, H_{\mu \nu}$ are Lorentz violating coefficients which arise from nonzero vacuum expectation values of tensor quantities and comprehensive describe effects of Lorentz violation on the behavior of particles coupling to these tensor fields. All of coefficients can be constrained as the real numbers if the action (11) is hermitian. In generally, they are functions of position. The two terms involving the couplings $a_{\mu}$ and $b_{\mu}$ are CPT odd, which have been extensively studied in connection with Lorentz- and CPT-violating probing experiments including comparative studies of cyclotron frequencies of trapped-atoms [17], clock comparison tests [18], spectroscopic comparison of hydrogen and antihydrogen 19], analysis of muon anomalous magnetic moment [20], study of macroscopic samples of spin-polarized solids [21], and so on.

On the other hand, black hole is another interesting object in the modern fundamental physics. It is believed widely that the study of black hole may lead to a deeper understanding of the relationship among the general relative theory, quantum mechanics, thermodynamics and statistics. This means that black hole physics play an important role in the fundamental physics. However, at present whether black holes exist in our universe or not is still unclear. A recent investigation shows that quasinormal modes can provide a direct way to identify black hole existence in our universe because that they carry the characteristic information of black holes [23] [24]. Moreover, it is also found that quasinormal modes have a close connection with the AdS/CFT correspondence [25] [26] [27] and the loop quantum gravity [28||29|. Thus, the study of quasinormal modes in black hole spacetimes has become appealing in recent years [30]-[44].

Since both Lorentz violation and the quasinormal modes are hot topics in physics at present, it is natural to raise a question whether Lorentz violation affects the quasinormal modes of black holes. From the action (11), we can obtain that due to presence of Lorentz violating coefficients Dirac equation must be modified and then its quasinormal frequencies in the black hole spacetimes should be changed. However, to my best knowledge it is still an open question how Lorentz violation affects the properties of quasinormal modes in the background of black hole spacetimes. Obviously, different Lorentz violating coefficients (i.e. different types of breaking of Lorentz symmetries) have different effects on quasinormal modes. In this paper, we just consider Dirac equation with a modified term containing Lorentz violating coefficient $b$ which arises from the nonzero vacuum 
expectation value of a special axial vector field $b_{\mu}[9][10]$ and calculate quasinormal modes of massless Dirac fields in the Schwarzschild black hole spacetime. Our result shows that in this case both fundamental and high overtones quasinormal frequencies depend on the Lorentz violating coefficient $b$.

The organization of this paper is as follows. In Sec.II, we derive the equation of massless Dirac field coupled with the special axial vector field $b_{\mu}$ in the Schwarzschild black hole spacetime. In Sec.III, we evaluate the fundamental overtones quasinormal frequencies of the Dirac perturbational field by using the third-order WKB approximation [45] [46] [47]. In Sec.IV, we adopt the monodromy technique [48] [49] and study the high-damped quasinormal frequencies. The last section is devoted to a summary.

\section{THE DIRAC EQUATION WITH LORENTZ VIOLATION ASSOCIATED WITH AN AXIAL-VECTOR $b_{\mu}$ FIELD}

According to the variation of $\bar{\psi}$ in the action (10), we find that the massless Dirac equation only containing the CPT and Lorentz covariance breaking kinetic term associated with an axial-vector $b_{\mu}$ field in the curve spacetime can be expressed as

$$
\left[i \gamma^{a} e_{a}{ }^{\mu}\left(\partial_{\mu}+\Gamma_{\mu}\right)-b_{\mu} e_{a}{ }^{\mu} \gamma_{5} \gamma^{a}\right] \Psi=0
$$

where

$$
\gamma^{0}=\left(\begin{array}{cc}
I & 0 \\
0 & -I
\end{array}\right), \quad \gamma^{i}=\left(\begin{array}{cc}
0 & \sigma^{i} \\
-\sigma^{i} & 0
\end{array}\right), \quad \gamma_{5}=i \gamma^{0} \gamma^{1} \gamma^{2} \gamma^{3}=\left(\begin{array}{cc}
0 & I \\
I & 0
\end{array}\right)
$$

Since the Lorentz violation is very small, it is reasonable for us to assume that the axial-vector $b_{\mu}$ field does not

change the background metric. For convenience, we take $b_{\mu}$ as a non-zero timelike vector $\left(\frac{b}{r^{2}}, 0,0,0\right)$, where $b$ is a constant. In the Schwarzschild spacetime, the vierbein can be defined as

$$
e_{\mu}^{a}=\left(\sqrt{1-\frac{2 M}{r}}, \frac{1}{\sqrt{1-\frac{2 M}{r}}}, r, r \sin \theta\right) .
$$

Setting $\Psi=\left(1-\frac{2 M}{r}\right)^{-\frac{1}{4}}(\sin \theta)^{-\frac{1}{2}} \Phi$ and substituting Eq.(6) into Eq.(44), the Dirac equation (4) can be simplified as

$$
\left[\frac{\gamma^{0}}{\sqrt{1-\frac{2 M}{r}}} \frac{\partial}{\partial t}+\sqrt{1-\frac{2 M}{r}} \gamma^{1}\left(\frac{\partial}{\partial r}+\frac{1}{r}\right)+\frac{\gamma^{2}}{r} \frac{\partial}{\partial \theta}+\frac{\gamma^{3}}{r \sin \theta} \frac{\partial}{\partial \varphi}+\frac{b}{r^{2} \sqrt{1-\frac{2 M}{r}}} \gamma_{5} \gamma^{0}\right] \Phi=0 .
$$

If we define a tortoise coordinate

$$
r_{*}=r+2 M \ln \left(\frac{r}{2 M}-1\right),
$$

and the ansatz

$$
\Phi=\left(\begin{array}{c}
\frac{i G^{( \pm)}(r)}{r} \phi_{j m}^{ \pm}(\theta, \varphi) \\
\frac{F^{( \pm)}(r)}{r} \phi_{j m}^{\mp}(\theta, \varphi)
\end{array}\right) e^{-i \omega t}
$$


with

$$
\begin{aligned}
& \phi_{j m}^{+}=\left(\begin{array}{c}
\sqrt{\frac{j+m}{2 j}} Y_{l}^{m-1 / 2} \\
\sqrt{\frac{j-m}{2 j}} Y_{l}^{m+1 / 2}
\end{array}\right), \quad k=j+\frac{1}{2}, \quad j=l+\frac{1}{2}, \\
& \phi_{j m}^{-}=\left(\begin{array}{c}
\sqrt{\frac{j-m+1}{2 j+2}} Y_{l}^{m-1 / 2} \\
-\sqrt{\frac{j+m+1}{2 j+2}} Y_{l}^{m+1 / 2}
\end{array}\right), \quad k=-\left(j+\frac{1}{2}\right), \quad j=l-\frac{1}{2},
\end{aligned}
$$

we find that the case for $(+)$ and $(-)$ in the functions $F^{ \pm}$and $G^{ \pm}$can be put together and Eq.(7) can be rewritten as

$$
\frac{d}{d r_{*}}\left(\begin{array}{c}
G \\
F
\end{array}\right)+\left(\frac{k}{r} \sqrt{1-\frac{2 M}{r}}-\frac{b}{r^{2}}\right)\left(\begin{array}{cc}
I & 0 \\
0 & -I
\end{array}\right)\left(\begin{array}{c}
G \\
F
\end{array}\right)=\left(\begin{array}{cc}
0 & \omega \\
-\omega & 0
\end{array}\right)\left(\begin{array}{c}
G \\
F
\end{array}\right) .
$$

It is very easy to find the decoupled equations for variables $F$ and $G$ can be expressed as

$$
\begin{aligned}
& \frac{d^{2} F}{d r_{*}^{2}}+\left(\omega^{2}-V_{1}\right) F=0 \\
& \frac{d^{2} G}{d r_{*}^{2}}+\left(\omega^{2}-V_{2}\right) G=0
\end{aligned}
$$

with

$$
\begin{aligned}
V_{1,2} & = \pm \frac{d W}{d r_{*}}+W^{2} \\
& =\frac{|k| \Delta^{\frac{1}{2}}}{r^{4}}\left[|k| \Delta^{\frac{1}{2}} \mp(r-3 M)-2 b\right]+\frac{b^{2} \pm 2 b(r-2 M)}{r^{4}},
\end{aligned}
$$

where $W=\frac{k}{r} \sqrt{1-\frac{2 M}{r}}-\frac{b}{r^{2}}$ and $\Delta=r(r-2 M)$. It is obvious that the potentials $V_{1}$ and $V_{2}$ are related to the coefficient $b$ of Lorentz violations, which means that the Dirac quasinormal modes should depend on Lorentz violation. If we set $b=0$, Eqs.(12)-(14) give the results of general Dirac fields in the Schwarzschild black hole spacetime [37]. Moreover, it is well known that the potentials $V_{1}$ and $V_{2}$ possess the same spectra of quasinormal frequencies because that they are supersymmetric partners derived from the same superpotential $W$. In the following, we therefore just make use of Eq.(12) to evaluate the Dirac quasinormal frequencies and write $V_{1}$ as $V$.

\section{THE FUNDAMENTAL DIRAC QUASINORMAL MODES WITH LORENTZ VIOLATING COEFFICIENT $b_{\mu}$}

In order to study the relationship between quasinormal frequencies and the coefficient $b$, we can take $M=1$. Fig. 1 shows the variation of the effective potential with the coefficient $b$ of Lorentz violations for fixed $k=3$. From this figure we can find that as $b$ increases, the peak value of the potential barrier gets lower and the location of the peak $\left(r=r_{p}\right)$ moves along the right. 


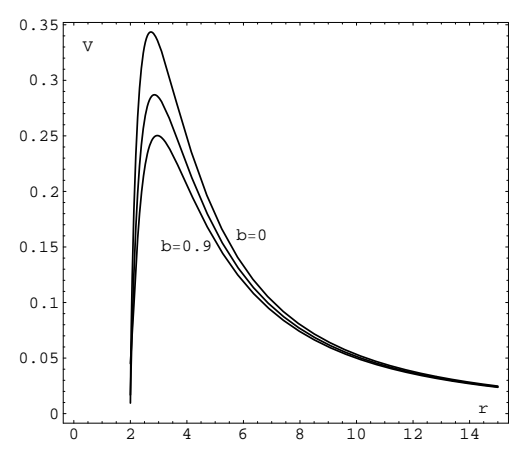

FIG. 1: Variation of the effective potential for the massless Dirac field $(k=3)$ with the coefficient $b$ of Lorentz violations

Let us now evaluate the fundamental quasinormal frequencies for the massless Dirac field with Lorentz violations by using the third-order WKB potential approximation, a numerical method devised by Schutz, Will and Iyer [45] [46] 47]. Due to its considerable accuracy for lower-lying modes, this method has been used extensively in evaluating quasinormal frequencies of various black holes. In this approximate method, the formula for the complex quasinormal frequencies $\omega$ is

$$
\omega^{2}=\left[V_{0}+\left(-2 V_{0}^{\prime \prime}\right)^{1 / 2} \Lambda\right]-i\left(n+\frac{1}{2}\right)\left(-2 V_{0}^{\prime \prime}\right)^{1 / 2}(1+\Omega),
$$

where

$$
\begin{aligned}
\Lambda & =\frac{1}{\left(-2 V_{0}^{\prime \prime}\right)^{1 / 2}}\left\{\frac{1}{8}\left(\frac{V_{0}^{(4)}}{V_{0}^{\prime \prime}}\right)\left(\frac{1}{4}+\alpha^{2}\right)-\frac{1}{288}\left(\frac{V_{0}^{\prime \prime \prime}}{V_{0}^{\prime \prime}}\right)^{2}\left(7+60 \alpha^{2}\right)\right\}, \\
\Omega & =\frac{1}{\left(-2 V_{0}^{\prime \prime}\right)}\left\{\frac{5}{6912}\left(\frac{V_{0}^{\prime \prime \prime}}{V_{0}^{\prime \prime}}\right)^{4}\left(77+188 \alpha^{2}\right)\right. \\
& -\frac{1}{384}\left(\frac{V_{0}^{\prime \prime \prime 2} V_{0}^{(4)}}{V_{0}^{\prime \prime 3}}\right)\left(51+100 \alpha^{2}\right)+\frac{1}{2304}\left(\frac{V_{0}^{(4)}}{V_{0}^{\prime \prime}}\right)^{2}\left(67+68 \alpha^{2}\right) \\
& \left.+\frac{1}{288}\left(\frac{V_{0}^{\prime \prime \prime} V_{0}^{(5)}}{V_{0}^{\prime \prime 2}}\right)\left(19+28 \alpha^{2}\right)-\frac{1}{288}\left(\frac{V_{0}^{(6)}}{V_{0}^{\prime \prime}}\right)\left(5+4 \alpha^{2}\right)\right\},
\end{aligned}
$$

and

$$
\alpha=n+\frac{1}{2}, \quad V_{0}^{(s)}=\left.\frac{d^{s} V}{d r_{*}^{s}}\right|_{r_{*}=r_{*}\left(r_{p}\right)},
$$

$n$ is overtone number.

Substituting the effective potential $V$ into the formula above, we can obtain the quasinormal frequencies for the Dirac fields with Lorentz violations. The fundament modes frequencies for $k=1 \sim 5$ are list in the Table 1 . From Fig.2, we find that for fixed $k$ the real part almost decrease linearly with the increase with $b$. Moreover, Fig. 3 tells us that the relationship between the magnitude of the imaginary parts and $b$ is more complex. For larger $k$, we find that they increase as $b$ increases, which means that presence of Lorentz violation makes Dirac oscillation damps more rapidly. 


\begin{tabular}{c|c|c|c|c|c}
\hline \hline$b$ & $\omega(k=1)$ & $\omega(k=2)$ & $\omega(k=3)$ & $\omega(k=4)$ & $\omega$ \\
\hline 0 & $0.176452-0.100109 \mathrm{i}$ & $0.378627-0.096542 \mathrm{i}$ & $0.573685-0.096320 \mathrm{i}$ & $0.767194-0.096276 \mathrm{i}$ & $0.960215-0.096256 \mathrm{i}$ \\
0.1 & $0.166462-0.100956 \mathrm{i}$ & $0.367984-0.096909 \mathrm{i}$ & $0.562847-0.096596 \mathrm{i}$ & $0.756264-0.096489 \mathrm{i}$ & $0.949237-0.096431 \mathrm{i}$ \\
0.2 & $0.157373-0.101275 \mathrm{i}$ & $0.357770-0.097175 \mathrm{i}$ & $0.552294-0.096829 \mathrm{i}$ & $0.745548-0.096681 \mathrm{i}$ & $0.938430-0.096592 \mathrm{i}$ \\
0.3 & $0.149036-0.101037 \mathrm{i}$ & $0.347972-0.097342 \mathrm{i}$ & $0.542020-0.097021 \mathrm{i}$ & $0.735043-0.096851 \mathrm{i}$ & $0.927792-0.096739 \mathrm{i}$ \\
0.4 & $0.141230-0.100325 \mathrm{i}$ & $0.338572-0.097408 \mathrm{i}$ & $0.532020-0.097174 \mathrm{i}$ & $0.724745-0.097000 \mathrm{i}$ & $0.917321-0.096873 \mathrm{i}$ \\
0.5 & $0.133666-0.099621 \mathrm{i}$ & $0.329548-0.097380 \mathrm{i}$ & $0.522288-0.097288 \mathrm{i}$ & $0.714652-0.097128 \mathrm{i}$ & $0.907015-0.096994 \mathrm{i}$ \\
0.6 & $0.126319-0.101000 \mathrm{i}$ & $0.320878-0.097266 \mathrm{i}$ & $0.512819-0.097363 \mathrm{i}$ & $0.704761-0.097234 \mathrm{i}$ & $0.896874-0.097101 \mathrm{i}$ \\
0.7 & $0.122890-0.114215 \mathrm{i}$ & $0.312532-0.097083 \mathrm{i}$ & $0.503605-0.097402 \mathrm{i}$ & $0.695069-0.097319 \mathrm{i}$ & $0.886894-0.097194 \mathrm{i}$ \\
0.8 & --- & $0.304478-0.096864 \mathrm{i}$ & $0.494639-0.097404 \mathrm{i}$ & $0.685573-0.097384 \mathrm{i}$ & $0.877074-0.097275 \mathrm{i}$ \\
0.9 & --- & $0.296676-0.096668 \mathrm{i}$ & $0.485914-0.097372 \mathrm{i}$ & $0.676269-0.097428 \mathrm{i}$ & $0.867413-0.097342 \mathrm{i}$ \\
1.0 & --- & $0.289080-0.096612 \mathrm{i}$ & $0.477423-0.097310 \mathrm{i}$ & $0.667154-0.097453 \mathrm{i}$ & $0.857907-0.097397 \mathrm{i}$ \\
\hline \hline
\end{tabular}

TABLE I: The fundamental overtones $(n=0)$ quasinormal frequencies of Dirac field with Lorentz violating kinetic term associated with an axial-vector $b_{\mu}$ in the Schwarzschild black hole spacetime for $k=1 \sim 5$.

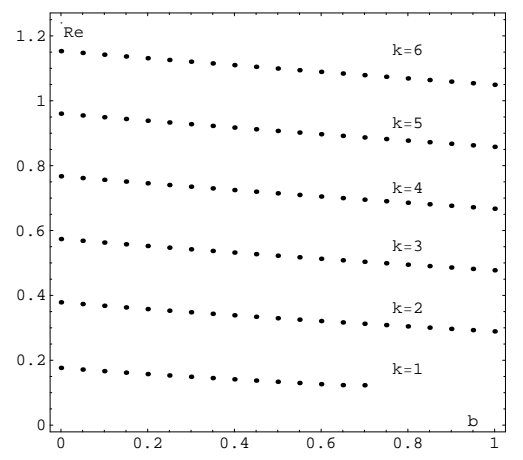

FIG. 2: Variation of the real parts of Dirac quasinormal frequencies with the coefficient $b$ of Lorentz violations.

\section{THE HIGH-DAMPED DIRAC QUASINORMAL MODES WITH LORENTZ VIOLATING COEFFICIENT $b_{\mu}$}

Motivated by Hod conjecture [28], a great deal of effort has been devoted to the study of the high-damped quasinormal modes in the different black hole spacetimes because that Hod's conjecture suggests that there maybe exist a connection between the high-damped quasinormal frequencies and quantum gravity. In this section, we adopt to the monodromy method[48] [49] and investigate the high-damped Dirac quasinormal modes with Lorentz violations in the Schwarzschild black hole spacetime. Our purpose is to probe whether Lorentz violations affects Hod conjecture. As in Ref.[48], after selecting the contour $L$ as shown in Fig.4, we can calculate the global monodromy around the contour $L$. In the neighborhood of the event horizon $r=2 M$, the effective potential $V$ and the solution of Eq.(12) can be approximated as

$$
V \sim \frac{b^{2}}{(2 M)^{4}}, \quad \phi(r) \sim e^{i \sqrt{\omega^{2}-\frac{b^{2}}{(2 M)^{4}}} z} .
$$

Since the only singularity of $\phi(r)$ or $e^{-i \omega z}$ inside the contour occurs at the point $r=2 M$. After a full clockwise round trip, $\phi(r)$ acquires a phase $e^{\frac{\pi \sqrt{\omega^{2}-\frac{b^{2}}{(2 M)^{4}}}}{\kappa}}$, while $e^{-i \omega z}$ acquires a phase $e^{-\frac{\pi \omega}{\kappa}}$. So the coefficient of $e^{-i \omega z}$ in the asymptotic of $\phi(r)$ must be multiplied by $e^{\frac{\pi\left(\omega+\sqrt{\left.\omega^{2}-\frac{b^{2}}{(2 M)^{4}}\right)}\right.}{\kappa}}$. 

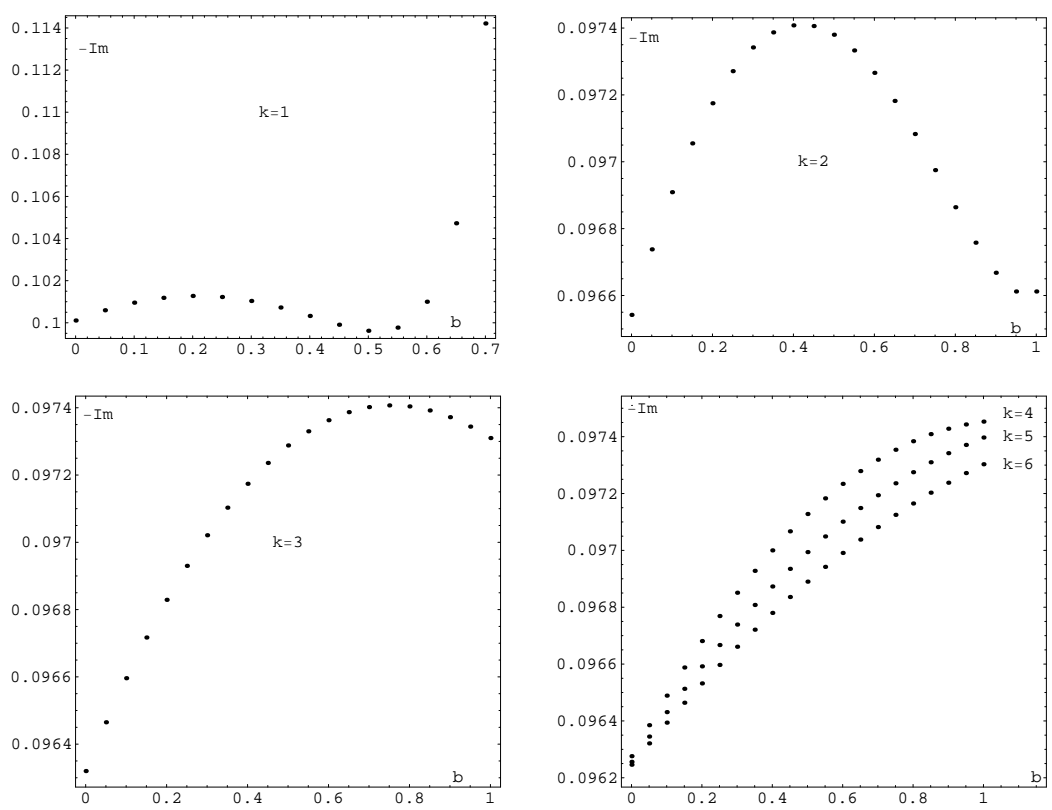

FIG. 3: Variation of the imaginary parts of Dirac quasinormal frequencies with the coefficient $b$ of Lorentz violations.

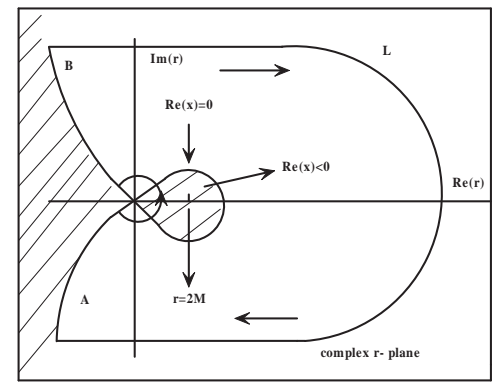

FIG. 4: The complex $r$-plane and the contour $L$. The regions with the hachures denote the area $\operatorname{Re}(x)<0$.

Moreover, we find that the behaviors of the tortoise coordinate $r_{*}$ and of the effective potential $V$ in Eq. (12) near the singular point $r=0$ are

$$
\begin{aligned}
r_{*} & \sim-\frac{r^{2}}{4 M}, \\
V & \sim \frac{b^{2}-4 M b}{r^{4}}=-\frac{1-j^{2}}{4 r_{*}^{2}},
\end{aligned}
$$

where $j=1-\frac{b}{2 M}$. As in Ref. [48], according to the local monodromy around the singular point $r=2 M$, we find that the monodromy around the contour $L$ must multiply the coefficient of $e^{-i \omega z}$ by a factor $-(1+2 \cos \pi j)$.

Comparing the local and global monodromy, we can obtain directly the high-damped quasinormal frequencies formula for Dirac fields with Lorentz violations in the Schwarzschild black hole spacetime

$$
e^{\frac{\pi\left[\omega+\sqrt{\omega^{2}-\frac{b^{2}}{(2 M)^{4}}}\right]}{\kappa}}=-\left[1+2 \cos \pi\left(1-\frac{b}{2 M}\right)\right], \quad n \rightarrow \infty .
$$

Where $\kappa$ is the surface gravity constant of the event horizon of the black hole. Comparing with mass $M$ of 
black hole, parameter $b$ is very small, thus the term $\frac{b^{2}}{(2 M)^{4}}$ in Eq.(19) can be neglected. The frequency formula for high-damped quasinormal modes can be further simplified as

$$
\omega=T_{H} \ln \left|\left[1+2 \cos \pi\left(1-\frac{b}{2 M}\right)\right]\right|-i 2 n \pi T_{H}, \quad n \rightarrow \infty
$$

where $T_{H}$ is the Hawking temperature of Schwarzschild black hole. It is obvious that Lorentz violating coefficient $b$ affects the high-damped Dirac quasinormal frequencies.

In the higher dimensional ( $D>4, D$ is the dimensions of spacetime ) Schwarzschild black hole spacetime, the axial vector field can be taken as $D$-component form $(\frac{b}{r^{D-2}}, \underbrace{0, \cdots, \cdots, 0}_{D-1})$. Similarly, we find that in this case the behaviors of the tortoise coordinate $r_{*}$ and of the effective potential $V$ near the singular point $r=0$ are

$$
\begin{aligned}
r_{*} & \sim-\frac{r^{D-2}}{2(D-2) M}, \\
V & \sim \frac{b^{2}-2(D-2) M b}{r^{2(D-2)}}=-\frac{1-j^{2}}{4 r_{*}^{2}}
\end{aligned}
$$

where $j=1-\frac{b}{(D-2) M}$. Repeating above operations, we can obtain that the high-damped Dirac quasinormal frequencies in the $D$ dimensional Schwarzschild black hole spacetime satisfy

$$
\omega=T_{H} \ln \left|1+2 \cos \pi\left[1-\frac{b}{(D-2) M}\right]\right|-i 2 n \pi T_{H}, \quad n \rightarrow \infty .
$$

It is shown that the asymptotic frequency formula of Dirac quasinormal modes can be also affected by the coefficient $b$ of Lorentz violation in the $D$ dimensional Schwarzschild black hole spacetime. When $b \rightarrow 0$, we find that the real parts of high-damped Dirac quasinormal frequencies in both cases become zero, which agrees with the result of Dirac field without Lorentz violations [43] [44]. According to Hod's idea [50], one can obtain that classical ringing frequencies with an asymptotically vanishing real part correspond to virtual quanta and the corresponding Dirac transitions in Lorentz invariance are quantum mechanically forbidden. However, from the formulas (20) and (22), we find that Dirac quantum transition is allowable in the Lorentz violation Frame. It implies that the emergence of Lorentz violation may change the quantum property of Dirac field.

\section{SUMMARY}

Adopting the third-order WKB approximation and monodromy methods, we investigated the quasinormal modes of Dirac fields with Lorentz violating term associated with a special axial-vector $b_{\mu}$ in the Schwarzschild black hole spacetime. We find that the coefficient $b$ of Lorentz violation affects Dirac quasionrmal frequencies. At fundamental overtone, the real part decreases linearly as the parameter $b$ increases. But the variation of the 
imaginary part with $b$ becomes more complex. For the larger multiple moment $k$, the magnitude of imaginary part increases with the increase of $b$, which means that presence of Lorentz violation makes Dirac field damps more rapidly. Since the imaginary part of quasinormal frequencies for large multiple number $k$ can be well approximated as [36] $\omega_{I} \sim \frac{-i}{3 \sqrt{3} G M}\left(n+\frac{1}{2}\right)$, the possible reason for our result is that the presence of the axial timelike vector field $b_{\mu}$ with nonzero vacuum expectation values may lead to the decrease of Newton's gravity constant $G$, which is possible in the Lorentz violating theories. Moreover, Dirac also supports that Newton's gravity constant $G$ decrease as increase of the age of universe. At high overtones, the real parts depend on Lorentz violating coefficient $b$. As $b \rightarrow 0$, we find the real part of high-damped Dirac quasinormal frequencies becomes zero, which consists with the result of Dirac field without Lorentz violations. Moreover, our result also shows that the emergence of Lorentz violation may change the quantum property of Dirac field. The effects of other Lorentz violating coefficients in the action (10) on quasinormal modes of black holes need to be investigated in the future.

\section{Acknowledgments}

We thank the referee for his/her quite useful and helpful comments and suggestions, which help deepen our understanding of Lorentz violation and quasinormal modes. This work was partially supported by NNSF of China, Ministry of Education of China and Shanghai Education Commission. R. K. Su's work was partially supported by the National Basic Research Project of China. S. B. Chen's work was partially supported by the Hunan Provincial Natural Science Foundation of China under Grant No.05JJ40012 and Scientific Research Fund of Hunan Normal University under Grant No.22040639. 
[1] Zatsepin G T and Kuzmin V A 1966 JETP Lett. 478

[2] Takeda M et al 1998 Phys. Rev. Lett. 811163

[3] Coleman S R and Glashow S L 1999 Phys. Rev. D 59116008

[4] Arkani-Hamed N, Cheng H C, Luty M A and Muko-hyama S 2004 JHEP 0405074

[5] Csaki C, Erlich J and Grojean C 2001 Nucl. Phys. B 604312

[6] Cline J M and L. Valcarcel, JHEP 0403032 (2004).

[7] Libanov M V and Rubakov V A 2005 Phys. Rev. D 72123503

[8] Kostelecky V A 2004 Phys. Rev. D 69105009

[9] Colladay D and Kostelecky V A 1997 Phys. Rev. D 556760

[10] Colladay D and Kostelecky V A 1998 Phys. Rev. D 58116002

[11] Bluhm R and Kostelecky V A 2005 Phys. Rev. D 71065008

[12] Kanno S and Soda J 2006 Phys. Rev. D74 063505

[13] Alfaro J, Andrianov A A, Cambiaso M, Giacconi P and Soldati R 2006 Phys. Lett. B 639 586-590

[14] Belich H, Costa-Soares T, Ferreira Jr M M, Helayel-Neto J A and Mouchereck F M O 2006 Phys. Rev. D74 065009

[15] Henson J 2006 Macroscopic observables and Lorentz violation in discrete quantum gravity Preprint gr-qc/0604040

[16] Martinez M R and Piran T 2006 JCAP0604 006

[17] Bluhm R, Kostelecky V A and Russell N 1997 Phys. Rev. Lett.79 1432

[18] Bluhm R, Kostelecky V A, Lane C D and Russell N 2002 Phys. Rev. Lett. 88090801

[19] Kostelecky V A and Russell N 1999 Phys. Rev. Lett. 822254

[20] Bluhm R, Kostelecky V A and Lane C D 2000 Phys. Rev. Lett. 841098

[21] Bluhm R and Kostelecky V A 2000 Phys. Rev. Lett. 841381

[22] Ferreira Jr M M and Mouchereck F M O 2006 Influence of Lorentz- and CPT-violating terms on the Dirac equation Preprint hep-ph/0601018

[23] Chandrasekhar S and Detweiler S L 1975 Proc. R. Soc. London A 344441

[24] Regge T and Wheeler J A 1957 Phys. Rev. 1081063

[25] Witten E 1998 Adv. Theor. Math. Phys.2 253

[26] Maldacena J 1998 Adv. Theor. Math. Phys.2 231

[27] Kalyana S R and Sathiapalan B 1999 Mod Phys. Lett. A 142635

[28] Hod S 1998 Phy. Rev. Lett. 814293

[29] Dreyer O 2003 Phy. Rev. Lett. 90081301

[30] Cardoso V and Lemos J P S 2001 Phys. Rev. D 63124015

[31] Konoplya R A 2002 Phys. Rev. D 66084007

[32] Starinets A O 2002 Phys. Rev. D 66124013

[33] Setare M R 2003 Class. Quant. Grav. 21 1453; 2004 Phys. Rev. D 69044016

[34] Natario J and Schiappa R 2004 Adv. Theor. Math. Phys. 8 1001-1131

[35] Leaver E W Proc. R. Soc. Lond. A 402285 (1985) ; 1986 Phys. Rev. D 34384

[36] Ferrari V and Mashhoon B 1984 Phys. Rev. D30 295-304

[37] Cho H T 2003 Phys. Rev. D 68024003

[38] Shao C G, Wang B, Abdalla E and Su R K 2005 Phys. Rev. D 71044003

[39] Wang B, Lin C Y and Molina C 2004 Phys. Rev. D 70064025

[40] Du D P, Wang B and Su R K 2004 Phys. Rev. D 70064024

[41] Zhidenko A 2004 Class. Quant. Grav. 21273

[42] Jing J L 2004 Phys. Rev. D 69084009

[43] Cho H T 2006 Phys. Rev. D 73024019

[44] Jing J L 2004 Phys. Rev. D 70065004; 2005 Phys. Rev. D 71 124011; 2005 Phys. Rev. D 71124006

[45] Schutz B F and Will C M 1985 Astrophys. J. Lett. Ed. 291 L33

[46] Iyer S and Will C M 1987 Phys. Rev.D 353621

[47] Iyer S 1987 Phys. Rev. D 353632

[48] Motl L and Neitzke A 2003 Adv. Theor. Math. Phys. 7307

[49] Andersson N 1991 Class. Quantum Grav. 10 L61

[50] Hod S and Keshet U 2006 Phys. Rev. D 73024003 